\title{
Breast Cancer Screening
}

National Cancer Institute

\section{Source}

National Cancer Institute. Breast Cancer Screening. NCI Thesaurus. Code C15546.

Early detection of breast cancer involving asymptomatic women. Generally done in the population with higher risk, due to environmental or genetic factors. 